\title{
Some properties for a subfunction associated with the stationary Schrödinger operator in a cone
}

\section{Pinhong Long and Guantie Deng*}

\section{"Correspondence:} denggt@bnu.edu.cn School of Mathematical Science, Laboratory of Mathematics and Complex Systems, Beijing Normal University, Beijing, 100875, P.R. China

\begin{abstract}
For a subfunction $u$ associated with the stationary Schrödinger operator, which is dominated on the boundary by a certain function on a cone, we correct Theorem 1 in (Qiao and Deng in Glasg. Math. J. 53(3):599-610, 2011). Then by the theorem we generalize some theorems of Phragmén-Lindelöf type for a subfunction in a cone. Meanwhile, we obtain some results about the existence of solutions of the Dirichlet problem associated with the stationary Schrödinger operator in a cone and about the type of their uniqueness.
\end{abstract}

MSC: Primary 31B05

Keywords: stationary Schrödinger operator; Poisson a-integral; subfunction; cone

\section{Introduction and main results}

To begin with, let us agree to some basic conventions. As usual, let $\mathbf{S}$ be an open set in $\mathbf{R}^{n}(n \geq 2)$, where $\mathbf{R}^{n}$ is the $n$-dimensional Euclidean space. The boundary and the closure of $\mathbf{S}$ are denoted by $\partial \mathbf{S}$ and $\overline{\mathbf{S}}$, respectively. Let $P=\left(X, x_{n}\right)$, where $X=\left(x_{1}, x_{2}, \ldots, x_{n-1}\right)$, and let $|P|$ be the Euclidean norm of $P$ and $|P-Q|$ be the Euclidean distance of two points $P$ and $Q$ in $\mathbf{R}^{n}$. The unit sphere and the upper half unit sphere are denoted by $\mathbf{S}^{n-1}$ and $\mathbf{S}_{+}^{n-1}$, respectively. For simplicity, the point $(1, \Theta)$ on $\mathbf{S}^{n-1}$ and the set $\{\Theta ;(1, \Theta) \in \Omega\}$ for a set $\Omega, \Omega \subset \mathbf{S}^{n-1}$ are often identified with $\Theta$ and $\Omega$, respectively. For $\Xi \subset \mathbf{R}_{+}$and $\Omega \subset \mathbf{S}^{n-1}$, the set $\left\{(r, \Theta) \in \mathbf{R}^{n} ; r \in \Xi,(1, \Theta) \in \Omega\right\}$ in $\mathbf{R}^{n}$ is simply denoted by $\Xi \times \Omega$. In particular, the half-space $\mathbf{R}_{+} \times \mathbf{S}_{+}^{n-1}=\left\{\left(X, x_{n}\right) \in \mathbf{R}^{n} ; x_{n}>0\right\}$ will be denoted by $\mathbf{T}_{n}$. By $C_{n}(\Omega)$, we denote the set $\mathbf{R}_{+} \times \Omega$ in $\mathbf{R}^{n}$ with the domain $\Omega$ on $\mathbf{S}^{n-1}$ and call it a cone. For an interval $I \subset$ $\mathbf{R}_{+}$and $\Omega \subset \mathbf{S}^{n-1}$, set $C_{n}(\Omega ; I)=I \times \Omega, S_{n}(\Omega ; I)=I \times \partial \Omega$ and $C_{n}(\Omega ; r)=C_{n}(\Omega) \cap S_{r}$. By $S_{n}(\Omega)$ we denote $S_{n}(\Omega ;(0,+\infty))$, which is $\partial C_{n}(\Omega)-\{O\}$. Furthermore, we denote by $d S_{r}$ the $(n-1)$-dimensional volume elements induced by the Euclidean metric on $S_{r}$. For $P \in$ $\mathbf{R}^{n}$ and $r>0$, let $B(P, r)$ denote the open ball with center at $P$ and radius $r$ in $\mathbf{R}^{n}$, then $S_{r}=\partial B(O, r)$.

We introduce the system of spherical coordinates $(r, \Theta), \Theta=\left(\theta_{1}, \theta_{2}, \ldots, \theta_{n-1}\right)$ for $P=$ $\left(X, x_{n}\right)$ in $\mathbf{R}^{n}$ via the following formulas:

$$
x_{1}=r \prod_{j=1}^{n-1} \sin \theta_{j} \quad(n \geq 2), \quad x_{n}=r \cos \theta_{1}
$$

๑ 2012 Long and Deng; licensee Springer. This is an Open Access article distributed under the terms of the Creative Commons Attribution License (http://creativecommons.org/licenses/by/2.0), which permits unrestricted use, distribution, and reproduction in any medium, provided the original work is properly cited. 
and if $n \geq 3$,

$$
x_{n-k+1}=r \cos \theta_{k} \prod_{j=1}^{k-1} \sin \theta_{j} \quad(2 \leq k \leq n-1),
$$

where $0 \leq r<\infty, 0 \leq \theta_{j} \leq \pi(1 \leq j \leq n-2)$ and $-\frac{\pi}{2} \leq \theta_{n-1} \leq \frac{3 \pi}{2}$.

Relative to the system of spherical coordinates, the Laplace operator $\Delta$ may be written

$$
\Delta=\frac{n-1}{r} \frac{\partial}{\partial r}+\frac{\partial^{2}}{\partial r^{2}}+\frac{\Delta^{n}}{r^{2}}
$$

where the explicit form of the Beltrami operator $\Delta^{*}$ is given by Azarin (see [1]).

For an arbitrary domain $D$ in $\mathbf{R}^{n}$, $\mathcal{A}_{D}$ denotes the class of non-negative radial potentials $a(P)($ i.e., $0 \leq a(P)=a(r)$ for $P=(r, \Theta) \in D)$ such that $a \in L_{\text {loc }}^{b}(D)$ with some $b>n / 2$ if $n \geq 4$ and with $b=2$ if $n=2$ or $n=3$.

If $a \in \mathcal{A}_{D}$, the stationary Schrödinger operator with a potential $a(\cdot)$,

$$
\mathcal{L}_{a}=-\Delta+a(\cdot) I
$$

can be extended in the usual way from the space $C_{0}^{\infty}(D)$ to an essentially self-adjoint operator on $L^{2}(D)$, where $\Delta$ is the Laplace operator and $I$ is the identical operator (see Reed and Simon [2], Chapter 13). Furthermore, $\mathcal{L}_{a}$ has a Green $a$-function $G_{D}^{a}(\cdot, \cdot)$. Here $G_{D}^{a}(\cdot, \cdot)$ is positive on $D$ and its inner normal derivative $\partial G_{D}^{a}(\cdot, Q) / \partial n_{Q} \geq 0$, where $\partial / \partial n_{Q}$ denotes the differentiation at $Q$ along the inward normal into $D$. We denote this derivative by $P I_{D}^{a}(\cdot, \cdot)$, which is called the Poisson $a$-kernel with respect to $D$. There is an inequality between the Green $a$-function $G_{D}^{a}(\cdot, \cdot)$ and that of the Laplacian, hereafter denoted by $G_{D}^{0}(\cdot, \cdot)$. It is well known that for any potential $a(\cdot) \geq 0$,

$$
G_{D}^{a}(\cdot, \cdot) \leq G_{D}^{0}(\cdot, \cdot)
$$

The inverse inequality is much more elaborate when $D$ is a bounded domain in $\mathbf{R}^{n}$. For a bounded domain $D$ in $\mathbf{R}^{n}$, Cranston, Fabes and Zhao (see [3], the case $n=2$ is implicitly contained in [4]) have proved

$$
G_{D}^{a}(\cdot, \cdot) \geq M(D) G_{D}^{0}(\cdot, \cdot),
$$

where $M(D)=M(D, a)$ is positive and independent of points in $D$. If $a=0$, obviously $M(D) \equiv 1$.

Suppose that a function $u \not \equiv-\infty$ is upper semi-continuous in $D . u \in[-\infty,+\infty)$ is called a subfunction of the Schrödinger operator $\mathcal{L}_{a}$ if the generalized mean-value inequality

$$
u(P) \leq \int_{S(P, \rho)} u(Q) \frac{\partial G_{B(P, \rho)}^{a}(P, Q)}{\partial n_{Q}} d \sigma(Q)
$$

is satisfied at each point $P \in D$ with $0<\rho<\inf _{Q \in \partial D}|P-Q|$, where $S(P, \rho)=\partial B(P, \rho)$, $G_{B(P, \rho)}^{a}(P, \cdot)$ is the Green $a$-function of $\mathcal{L}_{a}$ in $B(P, \rho)$ and $d \sigma(\cdot)$ is the surface area element on $S(P, \rho)$. 
Denote the class of subfunctions in $D$ by $\operatorname{SbH}(a, D)$. If $-u \in \operatorname{SbH}(a, D)$, we call $u$ a superfunction and denote the class of superfunctions by $\operatorname{SpH}(a, D)$. If a function $u$ is both subfunction and superfunction, clearly, it is continuous and called an $a$-harmonic function associated with the operator $\mathcal{L}_{a}$. The class of $a$-harmonic functions is denoted by $H(a, D)=\operatorname{SbH}(a, D) \cap \operatorname{SpH}(a, D)$. In terminology we follow Levin and Kheyfits (see [5] or [6]). From now on, we always assume $D=C_{n}(\Omega)$. For the sake of brevity, we shall write $G_{\Omega}^{a}(\cdot, \cdot)$ instead of $G_{C_{n}(\Omega)}^{a}(\cdot, \cdot), P I_{\Omega}^{a}(\cdot, \cdot)$ instead of $P I_{C_{n}(\Omega)}^{a}(\cdot, \cdot), S p H(a)$ (resp. $\left.S b H(a)\right)$ instead of $\operatorname{SpH}\left(a, C_{n}(\Omega)\right)$ (resp. $\left.\operatorname{SbH}\left(a, C_{n}(\Omega)\right)\right)$ and $H(a)$ instead of $H\left(a, C_{n}(\Omega)\right)$.

For positive functions $h_{1}$ and $h_{2}$, we say that $h_{1} \lesssim h_{2}$ if $h_{1} \leq M h_{2}$ for some constant $M>0$. If $h_{1} \lesssim h_{2}$ and $h_{2} \lesssim h_{1}$, we say that $h_{1} \approx h_{2}$.

Let $\Omega$ be a domain on $S^{n-1}$ with a smooth boundary, and let $\lambda$ be the least positive eigenvalue for $-\Delta^{*}$ on $\Omega$ (see [7], p.41),

$$
\begin{aligned}
& \left(\Delta^{*}+\lambda\right) \varphi(\Theta)=0 \quad \text { on } \Omega, \\
& \varphi(\Theta)=0 \quad \text { on } \partial \Omega .
\end{aligned}
$$

The corresponding eigenfunction is denoted by $\varphi(\Theta)$ satisfying $\int_{\Omega} \varphi^{2}(\Theta) d S_{1}=1$. In order to ensure the existence of $\lambda$ and $\varphi(\Theta)$, we put a rather strong assumption on $\Omega$ : if $n \geq 3$, then $\Omega$ is a $C^{2, \alpha}$-domain $(0<\alpha<1)$ on $\mathbf{S}^{n-1}$ surrounded by a finite number of mutually disjoint closed hypersurfaces (e.g., see Gilbarg and Trudinger [8], pp.88-89 for the definition of $C^{2, \alpha}$-domain). We denote the non-decreasing sequence of positive eigenvalues of (1.5) by $\{\lambda(\Omega, k)\}_{k=1}^{\infty}$. In the expression, we write $\lambda(\Omega, k)$ the same number of times as the dimension of the corresponding eigenspace. When the normalized eigenfunction corresponding to $\lambda(\Omega, k)$ is denoted by $\varphi_{k}(\Theta)$, the set of sequential eigenfunctions corresponding to the same value of $\lambda(\Omega, k)$ in the sequence $\left\{\varphi_{k}(\Theta)\right\}_{k=1}^{\infty}$ makes an orthonormal basis for the eigenspace of the eigenvalue $\lambda(\Omega, k)$. Hence, for each $\Omega \subset \mathbf{S}^{n-1}$, there is a sequence $\left\{k_{i}\right\}$ of positive integers such that $k_{1}=1, \lambda\left(\Omega, k_{i}\right)<\lambda\left(\Omega, k_{i+1}\right)$,

$$
\lambda\left(\Omega, k_{i}\right)=\lambda\left(\Omega, k_{i}+1\right)=\lambda\left(\Omega, k_{i}+2\right)=\cdots=\lambda\left(\Omega, k_{i+1}-1\right)
$$

and $\left\{\varphi_{k_{i}}(\Theta), \varphi_{k_{i}+1}(\Theta), \ldots, \varphi_{k_{i+1}-1}(\Theta)\right\}$ is an orthonormal basis for the eigenspace of the eigenvalue $\lambda\left(\Omega, k_{i}\right)(i=1,2,3, \ldots)$. It is well known that $k_{2}=2$ and $\varphi_{1}(\Theta)>0$ for any $\Theta \in \Omega$ (see Courant and Hilbert [9]). For the case $\Omega=\mathbf{S}_{+}^{n-1}(n=2,3,4, \ldots), k_{i}=i(i=1,2,3, \ldots)$ when $n=2$, and the situation is more complicated when $n \geq 3$ (see the Remark in [10] for the detailed information). For a domain $\Omega$ and the sequence $\left\{k_{i}\right\}$ mentioned above, by $I\left(k_{l}\right)$ we denote the set of all positive integers less than $k_{l}(k=1,2,3, \ldots)$. In spite of the fact that $I\left(k_{1}\right)=\phi$, the summation over $I\left(k_{1}\right)$ of a function $S(k)$ of a variable $k$ is used by promising

$$
\sum_{k \in I\left(k_{1}\right)} S(k)=0
$$

If $\Omega$ is an $(n-1)$-dimensional compact Riemannian manifold with its boundary to be sufficiently regular, we see that

$$
\lambda(\Omega, k) \sim A(\Omega, n) k^{\frac{2}{n-1}} \quad(k \rightarrow \infty)
$$


(e.g., see Cheng and $\mathrm{Li}[11])$ and

$$
\sum_{\lambda(\Omega, k) \leq x}\left\{\varphi_{k}(\Theta)\right\}^{2} \sim B(\Omega, n) x^{\frac{n-1}{2}} \quad(x \rightarrow \infty)
$$

uniformly with respect to $\Theta$ (e.g., see Minakshisundaram and Pleijel [12] or Essén and Lewis [13], p.120 and pp.126-128), where $A(\Omega, n)$ and $B(\Omega, n)$ are both constants depending on $\Omega$ and $n$. Hence, there exist two positive $M_{1}, M_{2}$ such that

$$
M_{1} k^{\frac{2}{n-1}} \leq \lambda(\Omega, k) \quad(k=1,2,3, \ldots)
$$

and

$$
\left|\varphi_{k}(\Theta)\right| \leq M_{2} k^{\frac{1}{2}} \quad(\Theta \in \Omega, k=1,2,3, \ldots) .
$$

Solutions of an ordinary differential equation

$$
-Q^{\prime \prime}(r)-\frac{n-1}{r} Q^{\prime}(r)+\left(\frac{\lambda}{r^{2}}+a(r)\right) Q(r)=0 \quad(0<r<\infty)
$$

are known (see [14] for more references) when the potential $a \in \mathcal{A}_{D}$. We know equation (1.11) has a fundamental system of positive solutions $\{V, W\}$ such that $V$ is non-decreasing with

$$
0 \leq V(0+) \leq V(r) \quad \text { as } r \rightarrow+\infty
$$

and $W$ is monotonically decreasing with

$$
+\infty=W(0+)>W(r) \searrow 0 \quad \text { as } r \rightarrow+\infty .
$$

We remark that both $V(r, k) \varphi_{k}(\Theta)$ and $W(r, k) \varphi_{k}(\Theta)(k=1,2,3, \ldots)$ are $a$-harmonic on $C_{n}(\Omega)$ and vanish continuously on $S_{n}(\Omega)$, where $V(r, k)$ and $W(r, k)$ are the solutions of equation (1.11) with $\lambda=\lambda(\Omega, k)$.

Let $\mathscr{B}_{D}$ be the class of the potentials $a \in \mathcal{A}_{D}$ such that

$$
\lim _{r \rightarrow \infty} r^{2} a(r)=\kappa \in[0, \infty), \quad r^{-1}\left|r^{2} a(r)-\kappa\right| \in L(1, \infty) .
$$

When $a \in \mathscr{B}_{D}$, the (super)subfunctions are continuous (e.g., see [15]). In the rest of paper, we assume that $a \in \mathcal{B}_{D}$ and we suppress this assumption for simplicity.

Denote

$$
\iota_{\kappa}^{ \pm}=\frac{2-n \pm \sqrt{(n-2)^{2}+4(\kappa+\lambda)}}{2} .
$$

When $a \in \mathscr{B}_{D}$, the solutions $V(r), W(r)$ to equation (1.11) normalized by $V(1)=W(1)=1$ have the asymptotic (see [8])

$$
V(r) \approx r^{\iota_{\kappa}^{+}}, \quad W(r) \approx r^{\iota_{\kappa}^{-}}, \quad \text { as } r \rightarrow \infty
$$


and

$$
\chi=\iota_{\kappa}^{+}-\iota_{\kappa}^{-}=\sqrt{(n-2)^{2}+4(\kappa+\lambda)}, \quad \chi^{\prime}=\left.\omega(V(r), W(r))\right|_{r=1},
$$

where $\chi^{\prime}$ is their Wronskian at $r=1$.

Remark 1.1 If $a=0$ and $\Omega=\mathbf{S}_{+}^{n-1}, \iota_{0}^{+}=1, \iota_{0}^{-}=1-n$ and $\varphi(\Theta)=\left(2 n s_{n}^{-1}\right)^{1 / 2} \cos \theta_{1}$, where $s_{n}$ is the surface area $2 \pi^{n / 2}\{\Gamma(n / 2)\}^{-1}$ of $\mathbf{S}^{n-1}$.

Let $u(r, \Theta)$ be a function on $C_{n}(\Omega)$. We introduce $M_{u}(r)=M(r, u)=\sup _{\Theta \in \Omega} u(r, \Theta), u^{+}=$ $\max \{u, 0\}$ and $u^{-}=\max \{-u, 0\}$.

We say that $u(P)(P=(r, \Theta))$ satisfies the Phragmén-Lindelöf boundary condition on $S_{n}(\Omega)$, namely

$$
\limsup _{P=(r, \Theta) \in C_{n}(\Omega), P \rightarrow Q^{*} \in S_{n}(\Omega)} u(P) \leq 0 .
$$

Let $F(P)=F(r, \Theta)$ be a function on $C_{n}(\Omega)$. For any given positive real number $r$, the integral

$$
\int_{\Omega} F(r, \Theta) \varphi(\Theta) d S_{1}
$$

is denoted by $N(F)(r)$, when it exists. For two non-negative integers $p$ and $q$, the finite or infinite limit

$$
\lim _{r \rightarrow \infty} \frac{N(F)(r)}{V\left(r, k_{p+1}\right)} \quad\left(\text { resp. } \lim _{r \rightarrow 0} \frac{N(F)(r)}{W\left(r, k_{q+1}\right)}\right)
$$

is denoted by $\mathcal{V}_{P}(F)\left(\right.$ resp. $\left.\mathcal{W}_{q}(F)\right)$, when it exists.

If $f(l)$ is a real finite-valued function defined on an interval $(0,+\infty)$, for any given $l_{1}, l_{2}$ $\left(0<l_{1}<l_{2}<\infty\right)$ and $l \in(0,+\infty)$, we have

$$
\mathcal{E}\left(l ; f, V, W, l_{1}, l_{2}\right)=\left|\begin{array}{ccc}
f(l) & V(l) & W(l) \\
f\left(l_{1}\right) & V\left(l_{1}\right) & W\left(l_{1}\right) \\
f\left(l_{2}\right) & V\left(l_{2}\right) & W\left(l_{2}\right)
\end{array}\right| \geq 0
$$

if and only if

$$
f(l) \leq \mathcal{F}\left(l ; f, V, W, l_{1}, l_{2}\right),
$$

where $\mathcal{F}\left(l ; f, V, W, l_{1}, l_{2}\right)$ has the following expression:

$$
\left\{\frac{W(l)}{W\left(l_{1}\right)} f\left(l_{1}\right)\left(\frac{V\left(l_{2}\right)}{W\left(l_{2}\right)}-\frac{V(l)}{W(l)}\right)+\frac{W(l)}{W\left(l_{2}\right)} f\left(l_{2}\right)\left(\frac{V(l)}{W(l)}-\frac{V\left(l_{1}\right)}{W\left(l_{1}\right)}\right)\right\}\left\{\frac{V\left(l_{2}\right)}{W\left(l_{2}\right)}-\frac{V\left(l_{1}\right)}{W\left(l_{1}\right)}\right\}^{-1} .
$$

We say that $f(l)$ is $(V, W)$-convex on $(0,+\infty)$ if $\&\left(l ; f, V, W, l_{1}, l_{2}\right) \geq 0\left(l_{1} \leq l \leq l_{2}\right)$ for any $l_{1}, l_{2}\left(0<l_{1}<l_{2}<+\infty\right)$. 
Remark 1.2 A function $f(l)$ is $(V, W)$-convex on $(0,+\infty)$ if and only if $W^{-1}(l) f(l)$ is a convex function of $W^{-1}(l) V(l)$ on $(0,+\infty)$ or, equivalently, if and only if $V^{-1}(l) f(l)$ is a convex function of $V^{-1}(l) W(l)$ on $(0,+\infty)$; refer to Dinghas [16] for the relevant properties of a convex function with respect to an $\mathrm{ODE}$.

The Poisson $a$-integral $P I_{\Omega}^{a}[g](P)$ of $g$ relative to $C_{n}(\Omega)$ is defined by

$$
P I_{\Omega}^{a}[g](P)=c_{n}^{-1} \int_{S_{n}(\Omega)} P I_{\Omega}^{a}(P, Q) g(Q) d \sigma_{Q}
$$

where

$$
P I_{\Omega}^{a}(P, Q)=\frac{\partial G_{\Omega}^{a}(P, Q)}{\partial n_{Q}}, \quad c_{n}= \begin{cases}2 \pi, & n=2, \\ (n-2) s_{n}, & n \geq 3,\end{cases}
$$

$\frac{\partial}{\partial n_{Q}}$ denotes the differentiation at $Q$ along the inward normal into $C_{n}(\Omega)$ and $d \sigma_{Q}$ is the surface area element on $S_{n}(\Omega)$.

For two non-negative integers $l, m$ and two points $P=(r, \Theta) \in C_{n}(\Omega)$ and $Q=(t, \Phi) \in$ $S_{n}(\Omega)$, we put

$$
\begin{aligned}
& \bar{V}\left(C_{n}(\Omega), l\right)(P, Q) \\
& \quad=\sum_{k \in I\left(k_{l+1}\right)} \frac{1}{\chi^{\prime}(1)} t^{-1} V(t, k) W(r, k) \varphi_{k}(\Theta) \frac{\partial \varphi_{k}(\Phi)}{\partial n_{\Phi}}
\end{aligned}
$$

and

$$
\begin{aligned}
& \underline{V}\left(C_{n}(\Omega), m\right)(P, Q) \\
& \quad=\sum_{k \in I\left(k_{m+1}\right)} \frac{1}{\chi^{\prime}(1)} t^{-1} W(t, k) V(r, k) \varphi_{k}(\Theta) \frac{\partial \varphi_{k}(\Phi)}{\partial n_{\Phi}} .
\end{aligned}
$$

We introduce two functions of $P \in C_{n}(\Omega)$ and $Q=(t, \Phi) \in S_{n}(\Omega)$ as follows:

$$
\bar{W}\left(C_{n}(\Omega), l\right)(P, Q)= \begin{cases}\bar{V}\left(C_{n}(\Omega), l\right)(P, Q) & (1 \leq t<\infty), \\ 0 & (0<t<1)\end{cases}
$$

and

$$
\underline{W}\left(C_{n}(\Omega), m\right)(P, Q)= \begin{cases}\underline{V}\left(C_{n}(\Omega), m\right)(P, Q) & (0<t<1) \\ 0 & (1 \leq t<\infty)\end{cases}
$$

The kernel $K\left(C_{n}(\Omega), l, m\right)(P, Q)$ with respect to $C_{n}(\Omega)$ is defined by

$$
\begin{aligned}
K\left(C_{n}(\Omega), l, m\right)(P, Q)= & c_{n}^{-1} \frac{\partial G_{C_{n}(\Omega)}^{a}}{\partial n_{\Phi}}(P, Q)-\bar{W}\left(C_{n}(\Omega), l\right)(P, Q) \\
& -\underline{W}\left(C_{n}(\Omega), m\right)(P, Q) .
\end{aligned}
$$


In fact

$$
K\left(C_{n}(\Omega), l, 0\right)(P, Q)=c_{n}^{-1} \frac{\partial G_{C_{n}(\Omega)}^{a}}{\partial n_{\Phi}}(P, Q)-\bar{W}\left(C_{n}(\Omega), l\right)(P, Q) \quad(l \geq 1)
$$

and

$$
K\left(C_{n}(\Omega), 0,0\right)(P, Q)=c_{n}^{-1} \frac{\partial G_{C_{n}(\Omega)}^{a}}{\partial n_{\Phi}}(P, Q) .
$$

Based on the elaborate research, Yoshida ([17] and [18]) has considered the subharmonic function defined on a cone or a cylinder which is dominated on the boundary by a certain function and generalized the classical Phragmén-Lindelöf theorem by making a harmonic majorant. Later Yoshida [19] proved the property of a harmonic function defined on a half-space which is represented by the generalized Poisson integral with a slowly growing continuous function on the boundary. In [20] or [10] Yoshida and Miyamoto generalized some theorems (from [19]) to the conical case and extended the results (from [17] and [18]) given particular solutions and a type of general solutions of the Dirichlet problem on a cone by introducing conical generalized Poisson kernels and Poisson integrals. On the other hand, Qiao and Deng [21] extended Yoshida's results (from [18]) to the situation for the stationary Schrödinger operator; for the relevant research on the stationary Schrödinger operator, we may refer to Bramanti [22], Kheyfits [23-30] and Levin et al. $[6,31]$. However, we find a falsehood in [21] and have to make a correction. In [5] or [6] we also know the Green function associated with the stationary Schrödinger operator. Dependent on the related statement above, we are to be concerned with the solutions of the Dirichlet problem for the stationary Schrödinger operator $\mathcal{L}_{a}$ on $C_{n}(\Omega)$ and with their growth properties. Furthermore, we note the existence of solutions of the Dirichlet problem for the stationary Schrödinger operator $\mathcal{L}_{a}$ in a cone and the type of their uniqueness. First of all, we start with the following result.

Theorem A Let $g(Q)$ be a continuous function on $S_{n}(\Omega)$ satisfying

$$
\int^{\infty} t^{-1} V(t)^{-1}\left(\int_{\partial \Omega}|g(t, \Phi)| d \sigma_{\Phi}\right) d t<\infty
$$

and

$$
\int_{0} t^{-1} W(t)^{-1}\left(\int_{\partial \Omega}|g(t, \Phi)| d \sigma_{\Phi}\right) d t<\infty
$$

Then the function $P I_{\Omega}^{a}[g](P)(P=(r, \Theta))$ satisfies

$$
\begin{aligned}
& P I_{\Omega}^{a}[g] \in C^{2}\left(C_{n}(\Omega)\right) \cap C^{0}\left(\overline{C_{n}(\Omega)}\right), \\
& \mathcal{L}_{a} P I_{\Omega}^{a}[g]=0 \quad \text { in } C_{n}(\Omega), \\
& P I_{\Omega}^{a}[g]=g \quad \text { on } \partial C_{n}(\Omega), \\
& \lim _{r \rightarrow \infty, P=(r, \Theta) \in C_{n}(\Omega)} V(r)^{-1} \varphi(\Theta)^{-1} P I_{\Omega}^{a}[g](P)=0
\end{aligned}
$$


and

$$
\lim _{r \rightarrow 0, P=(r, \Theta) \in C_{n}(\Omega)} W(r)^{-1} \varphi(\Theta)^{-1} P I_{\Omega}^{a}[g](P)=0 .
$$

Remark 1.3 As to Theorem 1 in the paper [21], the factor $\varphi_{\Omega}^{n-1}$ can be replaced with $\varphi_{\Omega}^{-1}$ such that it is true, that is, Theorem A corrects Theorem 1 (from [21]) which is a generalization for a result from Siegel and Talvila (see [32]). Moreover, as to Theorem A we may follow the proof procedure of Theorem 1 in [21].

Next, we state our main results as follows.

Theorem 1.4 Let $l, m$ be two non-negative integers and $g(Q)=g(t, \Phi)$ be a continuous function on $\partial C_{n}(\Omega)$ satisfying

$$
\int^{\infty} t^{-1} V\left(t, k_{l+1}\right)^{-1}\left(\int_{\partial \Omega}|g(t, \Phi)| d \sigma_{\Phi}\right) d t<\infty
$$

and

$$
\int_{0} t^{-1} W\left(t, k_{m+1}\right)^{-1}\left(\int_{\partial \Omega}|g(t, \Phi)| d \sigma_{\Phi}\right) d t<\infty .
$$

Then

$$
H\left(C_{n}(\Omega), l, m ; g\right)(P)=\int_{S_{n}(\Omega)} g(Q) K\left(C_{n}(\Omega), l, m\right)(P, Q) d \sigma_{Q}
$$

is a solution of the Dirichlet problem for the stationary Schrödinger operator on $C_{n}(\Omega)$ with g satisfying

$$
\mathcal{V}_{l}\left(\left|H\left(C_{n}(\Omega), l, m ; g\right)\right|\right)=\mathcal{W}_{m}\left(\left|H\left(C_{n}(\Omega), l, m ; g\right)\right|\right)=0
$$

Theorem 1.5 Let $l$ be a non-negative integer and $g(Q)=g(t, \Phi)$ be a continuous function on $\partial C_{n}(\Omega)$ satisfying

$$
\int^{\infty} t^{-1} V\left(t, k_{l+1}\right)^{-1}\left(\int_{\partial \Omega}|g(t, \Phi)| d \sigma_{\Phi}\right) d t<\infty
$$

Then

$$
H\left(C_{n}(\Omega), l, 0 ; g\right)(P)=\int_{S_{n}(\Omega)} g(Q) K\left(C_{n}(\Omega), l, 0\right)(P, Q) d \sigma_{Q}
$$

is a solution of the Dirichlet problem for the stationary Schrödinger operator on $C_{n}(\Omega)$ with $g$ satisfying

$$
\mathcal{V}_{l}\left(\left|H\left(C_{n}(\Omega), l, 0 ; g\right)\right|\right)=0
$$

Remark 1.6 When $m=0$, Theorem 1.4 is equal to Theorem 1.5. Since Theorem 1.4 may follow the proof for Theorem 1.5, for convenience, we only prove the latter. 
It is natural to ask if 0 in $(1.14)$ can be replaced with a general function $g\left(Q^{*}\right)$ on $S_{n}(\Omega)$. The following Theorem 1.7 gives an affirmative answer to this question. For related results, we refer the readers to the paper by Levin and Kheyfits (see [6], Section 3 or [5], Chapter 11).

Theorem 1.7 Let $p, q$ be two positive integers satisfying $p, q \geq 1$. Let $g\left(Q^{*}\right)$ be a continuous function on $S_{n}(\Omega)$ satisfying (1.25) and (1.26) and $u(P)$ be a subfunction on $C_{n}(\Omega)$ such that

$$
\limsup _{P \in C_{n}(\Omega), P \rightarrow Q^{*} \in S_{n}(\Omega)} u(P) \leq g\left(Q^{*}\right) .
$$

Then all of the limits $\mathcal{V}_{p}(u), \mathcal{W}_{q}(u), \mathcal{V}_{p}\left(u^{+}\right)$and $\mathcal{W}_{q}\left(u^{+}\right)\left(-\infty<\mathcal{V}_{p}(u), \mathcal{W}_{q}(u) \leq+\infty, 0 \leq\right.$ $\left.\mathcal{V}_{p}\left(u^{+}\right), \mathcal{W}_{q}\left(u^{+}\right) \leq+\infty\right)$ exist. Moreover, when

$$
\begin{aligned}
& \mathcal{V}_{p}\left(u^{+}\right)<+\infty \text { and } \mathcal{W}_{q}\left(u^{+}\right)<+\infty, \\
& u(P) \leq P I_{\Omega}^{a}[g](P)+\sum_{k \in I\left(k_{p+1}\right)} \mathcal{A}_{u}(k) V(r, k) \varphi_{k}(\Theta) \\
&+\sum_{k \in I\left(k_{q+1}\right)} \mathcal{B}_{u}(k) W(r, k) \varphi_{k}(\Theta)
\end{aligned}
$$

for any $P=(r, \Theta) \in C_{n}(\Omega)$, where $\mathcal{A}_{u}(k)\left(k=1,2, \ldots, k_{p+1}-1\right)$ and $\mathcal{B}_{u}(k)\left(k=1,2, \ldots, k_{q+1}-1\right)$ are all constants.

As an application of Theorems A and 1.7, we obtain the following result.

Theorem 1.8 Let $p, q$ be two positive integers satisfying $p, q \geq 1$. Let $g(Q)$ be defined as in Theorem 1.7 and $h(P)$ be any solution of the Dirichlet problem for the stationary Schrödinger operator $\mathcal{L}_{a}$ on $C_{n}(\Omega)$ with $g$. Then all of the limits $\mathcal{V}_{p}(h), \mathcal{W}_{q}(h), \mathcal{V}_{p}(|h|)$ and $\mathcal{W}_{q}(|h|)\left(-\infty<\mathcal{V}_{p}(h), \mathcal{W}_{q}(h) \leq+\infty, 0 \leq \mathcal{V}_{p}(|h|), \mathcal{W}_{q}(|h|) \leq+\infty\right)$ exist. Moreover, when

$$
\begin{aligned}
& \mathcal{V}_{p}(|h|)<+\infty \text { and } \mathcal{W}_{q}(|h|)<+\infty, \\
& h(P)= P I_{\Omega}^{a}[g](P)+\sum_{k \in I\left(k_{p+1}\right)} \mathcal{A}_{h}(k) V(r, k) \varphi_{k}(\Theta) \\
&+\sum_{k \in I\left(k_{q+1}\right)} \mathcal{B}_{h}(k) W(r, k) \varphi_{k}(\Theta)
\end{aligned}
$$

for any $P=(r, \Theta) \in C_{n}(\Omega)$, where $\mathcal{A}_{h}(k)\left(k=1,2, \ldots, k_{p+1}-1\right)$ and $\mathcal{B}_{h}(k)\left(k=1,2, \ldots, k_{q+1}-1\right)$ are all constants.

Remark 1.9 For $p=q=1$, Theorems 1.7 and 1.8 come from Qiao and Deng [21]. Furthermore, when $a=0$ and $p=q=1$, Theorems 1.7 and 1.8 are due to Yoshida (see [18], Theorems 2 and 3(II)). In fact, for $k \in I\left(k_{p+1}\right)$ we know $\mathcal{A}_{u}(k), \mathcal{B}_{u}(k)$ (or $\mathcal{A}_{h}(k), \mathcal{B}_{h}(k)$ ) are equal to the corresponding $\mathcal{V}(u), \mathcal{W}(u)$ (or $\mathcal{V}(h), \mathcal{W}(h))$, respectively. Without the potential function, we may refer to Yoshida (see [33]).

Theorem 1.10 Let $l, m$ be two non-negative integers and $p, q$ be two positive integers satisfying $p, q \geq 1$. Let $g(Q)$ be defined as in Theorem 1.7 satisfying (1.25) with land (1.26) with 
$m$, respectively. If $h(P)$ is any solution of the Dirichlet problem for the stationary Schrödinger operator $\mathcal{L}_{a}$ on $C_{n}(\Omega)$ with $g$ satisfying

$$
\begin{aligned}
\mathcal{V}_{p}\left(h^{+}\right) & =0 \quad \text { and } \quad \mathcal{W}_{q}\left(h^{+}\right)=0, \\
h(P)= & H\left(C_{n}(\Omega), l, m ; g\right)(P)+\sum_{k \in I\left(k_{p+1}\right)} \mathcal{A}_{h}(k) V(r, k) \varphi_{k}(\Theta) \\
& +\sum_{k \in I\left(k_{q+1}\right)} \mathcal{B}_{h}(k) W(r, k) \varphi_{k}(\Theta)
\end{aligned}
$$

for any $P=(r, \Theta) \in C_{n}(\Omega)$, where $\mathcal{A}_{h}(k)\left(k=1,2, \ldots, k_{p+1}-1\right)$ and $\mathcal{B}_{h}(k)\left(k=1,2, \ldots, k_{q+1}-1\right)$ are all constants.

Theorem 1.11 Let $l$ be a non-negative integer and $p$ be a positive integer satisfying $p \geq 1$. If $h(r, \Theta)$ is a generalized harmonic function on $C_{n}(\Omega)$ and continuous on $\overline{C_{n}(\Omega)}$ such that the restriction $h=\left.h\right|_{\partial C_{n}(\Omega)}$ of $h$ to $\partial C_{n}(\Omega)$ satisfies

$$
\int_{1}^{\infty} t^{-1} V\left(t, k_{l+1}\right)^{-1}\left(\int_{\partial \Omega_{n}}|h(t, \Phi)| d \sigma_{\Phi}\right) d t<\infty
$$

for some non-negative integer $l$, and for a positive integer $\widetilde{p}$

$$
\limsup _{r \rightarrow \infty} \frac{\log N\left(h^{+}\right)(r)}{\log V\left(r, k_{\tilde{p}+1}\right)}<\infty
$$

then, for some positive integer $p=\max \{l, \widetilde{p}\}$,

$$
h(P)=H\left(C_{n}(\Omega), l ; g\right)(P)+\sum_{k \in I\left(k_{p+1}\right)} \mathcal{A}_{h}(k) V(r, k) \varphi_{k}(\Theta)
$$

for any $P=(r, \Theta) \in C_{n}(\Omega)$, where $\mathcal{A}_{h}(k)\left(k=1,2, \ldots, k_{p+1}-1\right)$ are all constants.

Remark 1.12 If we take $a=0$, Theorems 1.10 and 1.11 are similar to Theorems 7 and 9 in [20], respectively. In [33] Yoshida and Miyamoto considered the case when $q=0, m=0$ and $a=0$ about Theorem 1.10 and gave the proof. In addition, with Theorem 1.10 we easily get the conclusion of Theorem 1.11, then we do not have to prove it.

\section{Some lemmas}

In our arguments, we need some important results and techniques, which result from [5, 6, 27, 34], and [1] (Lemma 4 and Remark).

\section{Lemma 2.1}

$$
\begin{aligned}
& P I_{\Omega}^{a}(P, Q) \approx t^{-1} V(t) W(r) \varphi(\Theta) \frac{\partial \varphi(\Phi)}{\partial n_{\Phi}} \\
& \left(\text { resp. } P I_{\Omega}^{a}(P, Q) \approx V(r) t^{-1} W(t) \varphi(\Theta) \frac{\partial \varphi(\Phi)}{\partial n_{\Phi}}\right)
\end{aligned}
$$


for any $P=(r, \Theta) \in C_{n}(\Omega)$ and any $Q=(t, \Phi) \in S_{n}(\Omega)$ satisfying $0<\frac{t}{r} \leq \frac{4}{5}\left(\right.$ resp. $\left.0<\frac{r}{t} \leq \frac{4}{5}\right)$. In addition,

$$
P I_{\Omega}^{0}(P, Q) \lesssim \frac{\varphi(\Theta)}{t^{n-1}} \frac{\partial \varphi(\Phi)}{\partial n_{\Phi}}+\frac{r \varphi(\Theta)}{|P-Q|^{n}} \frac{\partial \varphi(\Phi)}{\partial n_{\Phi}}
$$

for any $P=(r, \Theta) \in C_{n}(\Omega)$ and any $Q=(t, \Phi) \in S_{n}\left(\Omega ;\left(\frac{4}{5} r, \frac{5}{4} r\right)\right)$.

Lemma 2.2 Let $a \in \mathscr{B}_{D}$. For a non-negative integer $k_{l+1}$, we have

$$
\begin{aligned}
& \left|c_{n}^{-1} \frac{\partial G_{C_{n}(\Omega)}^{a}}{\partial n_{\Phi}}(P, Q)-\bar{W}\left(C_{n}(\Omega), l\right)(P, Q)\right| \\
& \quad \leq M\left(k_{l+1}, n, s\right) V\left(r, k_{l+1}\right) t^{-1} W\left(t, k_{l+1}\right) \varphi_{k}(\Theta) \frac{\partial \varphi_{k}(\Phi)}{\partial n_{\Phi}}
\end{aligned}
$$

for any $P=(r, \Theta) \in C_{n}(\Omega)$ and $Q=(t, \Phi) \in S_{n}(\Omega)$ satisfying $r \leq s t(0<s<1)$, where $M\left(k_{l+1}, n, s\right)$ is a constant dependent on $n, k_{l+1}$ and $s$.

Lemma 2.3 Let $g(Q)$ be a locally integrable and upper semicontinuous function on $\partial C_{n}(\Omega)$. Let $W(P, Q)$ be a function of $P \in C_{n}(\Omega)$ and $Q \in \partial C_{n}(\Omega)$ such that for any fixed $P \in C_{n}(\Omega)$ the function $W(P, Q)$ of $Q \in \partial C_{n}(\Omega)$ is a locally integrable function on $\partial C_{n}(\Omega)$. Put

$$
K(P, Q)=c_{n}^{-1} \frac{\partial G_{C_{n}(\Omega)}^{a}}{\partial n_{\Phi}}(P, Q)-W(P, Q) \quad\left(P \in C_{n}(\Omega), Q \in \partial C_{n}(\Omega)\right)
$$

Suppose that the following (I) and (II) are satisfied.

(I) For any $Q^{*} \in \partial C_{n}(\Omega)$ and any $\varepsilon>0$, there exist a neighborhood $U\left(Q^{*}\right)$ of $Q^{*}$ in $\mathbf{R}^{n}$ and a number $R(0<R<\infty)$ such that

$$
\int_{S_{n}(\Omega ;[R, \infty))}|g(Q) K(P, Q)| d \sigma_{\Phi}<\varepsilon
$$

for any $p=(r, \Theta) \in \partial C_{n}(\Omega) \cap U\left(Q^{*}\right)$

(II) For any $Q^{*} \in \partial C_{n}(\Omega)$ and any $R(0<R<\infty)$,

$$
\limsup _{P \rightarrow Q^{*}, P \in C_{n}(\Omega)} \int_{S_{n}(\Omega ;(0, R))}|g(Q) K(P, Q)| d \sigma_{\Phi}=0 .
$$

Then

$$
\limsup _{P \rightarrow Q^{*}, P \in C_{n}(\Omega)} \int_{S_{n}(\Omega)} g(Q) K(P, Q) d \sigma_{\Phi} \leq g\left(Q^{*}\right)
$$

for any $Q^{\prime \prime} \in \partial C_{n}(\Omega)$.

Remark 2.4 When $a=0$, Lemma 2.3 is due to Yoshida (see [10], Lemma 5). By (1.2), obviously we reduce to Yoshida's results. Therefore, we may omit the proof. 
Lemma 2.5 If $h(r, \Theta)$ is an a-harmonic function on $C_{n}(\Omega)$ vanishing continuously on $S_{n}(\Omega)$,

$$
\mathcal{E}\left(r ; N(h), V, W, r_{1}, r_{2}\right)=0
$$

for any $r_{1}, r_{2}\left(0<r_{1}<r_{2}<+\infty\right)$ and every $r(0<r<+\infty)$.

Lemma 2.6 If $f(l)$ is $(V, W)$-convex on $\left(0, d_{1}\right)\left(0<d_{1} \leq+\infty\right)$, then

$$
\beta=\lim _{l \rightarrow 0} \frac{f(l)}{W(l)} \quad(-\infty<\alpha \leq+\infty)
$$

exists. Further, if $\beta \leq 0, V^{-1}(l) f(l)$ is non-decreasing on $\left(0, d_{1}\right)$.

It is known that $C_{n}(\Omega)$ is regular, the Dirichlet problem for $\Delta$ and $\mathcal{L}_{a}$ is solvable in it (see [6]). Based on this fact, Lemmas 2.7, 2.8 and 2.9 could be derived from (1.2), (1.3), (1.12), Remark 1.2, Lemmas 2.3 and 2.5 with their means of proof essentially due to Yoshida (see [17], Theorems 3.1 and 5.1, and [18], Lemma 3).

Lemma 2.7 If $u(r, \Theta)$ is a subfunction on $C_{n}(\Omega)$ satisfying the Phragmén-Lindelöf boundary condition on $S_{n}(\Omega)$, then

$$
N(u)(r)>-\infty
$$

for $0<r<+\infty$ and $N(u)(r)$ is $(V, W)$-convex on $(0,+\infty)$. If there are three numbers $r_{1}, r_{2}$ and $r_{0}$ satisfying $0<r_{1}<r_{0}<r_{2}<+\infty$ such that

$$
\mathcal{E}\left(r_{0} ; N(u), V, W, r_{1}, r_{2}\right)=0 \text {, }
$$

we have that

(1) $\&\left(r ; N(u), V, W, r_{1}, r_{2}\right)=0\left(r_{1} \leq r \leq r_{2}\right)$;

(2) $u(r, \Theta)$ is an a-harmonic function on $C_{n}\left(\Omega ;\left(r_{1}, r_{2}\right)\right)$ and vanishes continuously on $S_{n}\left(\Omega ;\left(r_{1}, r_{2}\right)\right)$.

Lemma 2.8 Let $g(Q)$ be defined as in Theorem 1.7. Then $P I_{\Omega}^{a}[g](P)$ (resp. PI $\left.I_{\Omega}^{a}[|g|](P)\right)$ is an a-harmonic function on $C_{n}(\Omega)$ such that both of the limits $\mathcal{V}\left(P I_{\Omega}^{a}[g]\right)$ and $\mathcal{W}\left(P I_{\Omega}^{a}[g]\right)$ (resp. $\mathcal{V}\left(P I_{\Omega}^{a}[|g|]\right)$ and $\left.\mathcal{W}\left(P I_{\Omega}^{a}[|g|]\right)\right)$ exist, and

$$
\mathcal{V}\left(P I_{\Omega}^{a}[g]\right)=\mathcal{W}\left(P I_{\Omega}^{a}[g]\right)=0 \quad\left(\operatorname{resp} . \mathcal{V}\left(P I_{\Omega}^{a}[|g|]\right)=\mathcal{W}\left(P I_{\Omega}^{a}[|g|]\right)=0\right)
$$

Lemma 2.9 Let $u(P)$ be a subfunction on $C_{n}(\Omega)$ satisfying the Phragmén-Lindelöf boundary condition on $S_{n}(\Omega)$. If $(1.32)$ is satisfied for $p=1$ and $q=1$,

$$
u(P) \leq(\mathcal{V}(u) V(r)+\mathcal{W}(u) W(r)) \varphi(\Theta)
$$

for any $P=(r, \Theta) \in C_{n}(\Omega)$. 
By the Kelvin transformation (see [35], p.59), Lemmas 2.6 and 2.7, we immediately have the following result, which is due to Yoshida in the case $a=0$ (see [17], Theorem 3.3).

Lemma 2.10 Let $u(P)$ be defined as in Lemma 2.9. Then

(1) Both of the limits $\mathcal{V}(u)$ and $\mathcal{W}(u)(-\infty<\mathcal{V}(u), \mathcal{W}(u) \leq+\infty)$ exist.

(2) If $\mathcal{W}(u) \leq 0$, then $V^{-1}(r) N(u)(r)$ is non-decreasing on $(0,+\infty)$.

(3) If $\mathcal{V}(u) \leq 0$, then $W^{-1}(r) N(u)(r)$ is non-increasing on $(0,+\infty)$.

Lemma 2.11 Let $H(r, \Theta)$ be an a-harmonic function in $C_{n}(\Omega)$ vanishing continuously on $\partial C_{n}(\Omega)$, and $p, q$ be two positive integers. $h$ satisfies

$$
\mathcal{V}_{p}\left(h^{+}\right)=0 \text { and } \mathcal{W}_{q}\left(h^{+}\right)=0
$$

then

$$
h(P)=\sum_{k \in I\left(k_{p+1}\right)} \mathcal{A}_{h}(k) V(r, k) \varphi_{k}(\Theta)+\sum_{k \in I\left(k_{q+1}\right)} \mathcal{B}_{h}(k) W(r, k) \varphi_{k}(\Theta)
$$

for any $P=(r, \Theta) \in C_{n}(\Omega)$, where $\mathcal{A}_{u}(k)\left(k=1,2, \ldots, k_{p+1}-1\right)$ and $\mathcal{B}_{u}(k)\left(k=1,2, \ldots, k_{q+1}-1\right)$ are all constants.

Remark 2.12 When $q=0$ and $a=0$, Yoshida states the result in [10]. Later Qiao [34] proves Lemma 2.11 when $q=0$. Similarly, we may complete the proof of Lemma 2.11. Herein we leave out the detailed information for the proof.

\section{Proofs of the theorems}

Proof of Theorem 1.5 For any fixed $P \in C_{n}(\Omega)$, we take a number $R$ satisfying $s R>\max \{1, r\}$ $(0<s<1)$. Then from Lemma 2.2 and $(1.25)$

$$
\begin{aligned}
& \int_{S_{n}(\Omega ;[R, \infty))}\left|g(Q) K\left(C_{n}(\Omega), l, 0\right)(P, Q)\right| d \sigma_{\Phi} \\
& \quad=\int_{S_{n}(\Omega ;[R, \infty))}|g(Q)|\left|C_{n}^{-1} \frac{\partial G_{C_{n}(\Omega)}^{a}}{\partial n_{\Phi}}(P, Q)-\bar{W}\left(C_{n}(\Omega), l\right)(P, Q)\right| d \sigma_{\Phi} \\
& \quad \lesssim V\left(r, k_{l+1}\right) \varphi_{k}(\Theta) \int_{R}^{\infty} t^{-1} V\left(t, k_{l+1}\right)^{-1}\left(\int_{\partial \Omega}|g(t, \Phi)| d \sigma_{\Phi}\right) d t<\infty
\end{aligned}
$$

Then $H\left(C_{n}(\Omega), l, 0 ; g\right)(P)$ is absolutely convergent and finite for any $P \in C_{n}(\Omega)$. We remark that $K\left(C_{n}(\Omega), l, 0\right)(P, Q)$ is a harmonic function of $P \in C_{n}(\Omega)$ for any $Q \in C_{n}(\Omega)$. Thus, $H\left(C_{n}(\Omega), l, 0 ; g\right)(P)$ is a generalized harmonic function of $P \in C_{n}(\Omega)$.

Next, we consider the boundary behavior of $H\left(C_{n}(\Omega), l, 0 ; g\right)(P)$. To prove that

$$
\limsup _{P \rightarrow Q^{*}, P \in C_{n}(\Omega)} H\left(C_{n}(\Omega), l, 0 ; g\right)(P)=g\left(Q^{*}\right)
$$

for any $Q^{*} \in \partial C_{n}(\Omega)$, we may apply Lemma 2.3 to $g(Q)$ and $-g(Q)$ by putting

$$
W(P, Q)=\bar{W}\left(C_{n}(\Omega), l, 0\right)(P, Q)
$$


which is locally integrable on $C_{n}(\Omega)$ for any fixed $P \in C_{n}(\Omega)$. Let $\delta$ be a positive number and take $Q^{*}=\left(t^{*}, \Phi^{*}\right) \in \partial C_{n}(\Omega)$ and any $\varepsilon>0$. Then from (1.25) and (3.1) we can choose a number $R(s R>\max \{1, r\})(0<s<1)$ such that for any $P \in C_{n}(\Omega) \cap U_{\delta}\left(Q^{*}\right)$, where $U_{\delta}\left(Q^{*}\right)=$ $\left\{X \in \mathbf{R}^{n}:\left|X-Q^{*}\right|<\delta\right\}$ and $\delta$ is a positive number

$$
\int_{S_{n}(\Omega ;[R, \infty))}\left|g(Q) K\left(C_{n}(\Omega), l, 0\right)(P, Q)\right| d \sigma_{\Phi}<\varepsilon,
$$

which is (I) in Lemma 2.3 .

Because

$$
\lim _{\Theta \rightarrow \Phi^{*}} \varphi_{k}(\Theta)=0 \quad(k=1,2, \ldots)
$$

as $P=(r, \Theta) \rightarrow Q^{*}=\left(t^{*}, \Phi^{*}\right) \in \partial C_{n}(\Omega)$, we know that for any $Q^{*} \in \partial C_{n}(\Omega)$ and $Q \in \partial S_{n}(\Omega)$,

$$
\lim _{P \rightarrow Q^{*}, P \in C_{n}(\Omega)} \bar{W}\left(C_{n}(\Omega), l, 0\right)(P, Q)=0 .
$$

According to Lemma 2.3, we get the required results.

Next, we note the inequality

$$
N\left(\left|H\left(C_{n}(\Omega), l, 0 ; g^{+}\right)\right|\right)(r) \leq I_{1}(r)+I_{2}(r),
$$

where

$$
I_{1}(r)=\int_{\Omega}\left(\int_{S_{n}(\Omega ;[r, \infty))} g^{+}(Q)\left|K\left(C_{n}(\Omega), l, 0\right)(P, Q)\right| d \sigma_{Q}\right) \varphi_{k}(\Theta) d \sigma_{\Phi}
$$

and

$$
I_{2}(r)=\int_{\Omega}\left(\int_{S_{n}(\Omega ;[0, r))} g^{+}(Q)\left|K\left(C_{n}(\Omega), l, 0\right)(P, Q)\right| d \sigma_{Q}\right) \varphi_{k}(\Theta) d \sigma_{\Phi}
$$

for $P=(r, \Theta), 0<r<\infty$. For any positive number $\varepsilon$, from (1.25) we can take a sufficiently large number $r_{0}$ such that

$$
\int_{r}^{\infty} t^{-1} V\left(t, k_{l+1}\right)^{-1}\left(\int_{\partial \Omega}|g(t, \Phi)| d \sigma_{\Phi}\right) d t<\frac{\varepsilon}{L M} \quad\left(r>r_{0}\right),
$$

where $M$ is the constant in Lemma 2.2 and

$$
L=\int_{\Omega} \varphi_{k}(\Theta) d \sigma_{\Theta}
$$

Then from Lemma 2.2 we have

$$
\begin{aligned}
0 & \leq I_{1}(r) \leq L\left(k_{l+1}\right) M\left(n, k_{l+1}, s\right) V\left(r, k_{l+1}\right) \int_{r}^{\infty} t^{-1} V\left(t, k_{l+1}\right)^{-1}\left(\int_{\partial \Omega} g^{+}(t, \Phi) d \sigma_{\Phi}\right) d t \\
& \leq \varepsilon V\left(r, k_{l+1}\right) \quad\left(r>r_{0}\right)
\end{aligned}
$$

which gives $\mathcal{V}_{l}\left(I_{1}\right)=0$. 
Following this, we see the inequality

$$
I_{2}(r) \leq I_{21}(r)+I_{22}(r)
$$

where

$$
I_{21}(r)=\int_{\Omega}\left(\int_{S_{n}(\Omega ;[r, \infty))} g^{+}(Q) c_{n}^{-1} \frac{\partial G_{C_{n}(\Omega)}^{a}}{\partial n_{\Phi}}(P, Q) d \sigma_{Q}\right) \varphi_{k}(\Theta) d \sigma_{\Phi}
$$

and

$$
I_{22}(r)=\int_{\Omega}\left(\int_{S_{n}(\Omega ;[0, r))} g^{+}(Q) \bar{W}\left(C_{n}(\Omega), l\right)(P, Q) d \sigma_{Q}\right) \varphi_{k}(\Theta) d \sigma_{\Phi}
$$

for $P=(r, \Theta)$ and $r>1$. First, we know from (1.10) and (1.16) that if $l \geq 1$

$$
I_{22}(r) \lesssim \sum_{k \in I\left(k_{m+1}\right)} k V(r, k) \Psi_{k}(r) \quad(r>1)
$$

where

$$
\Psi_{k}(r)=\int_{1}^{r} t^{-1} V(t, k)^{-1}\left(\int_{\partial \Omega} g^{+}(t, \Phi) d \sigma_{\Phi}\right) d t \quad\left(r>1, k \in I\left(k_{m+1}\right)\right) .
$$

We claim that

$$
\Psi_{k}(r)=O\left(V\left(r, k_{l+1}\right) V(r, k)^{-1}\right) \quad\left(r>1, k \in I\left(k_{m+1}\right)\right) .
$$

First we note increasing $\Psi_{k}(r)$ and Lemma C.1 in [5], Chapter 13 or [6], then by (1.12) we get

$$
\Psi_{k}(r) V\left(r, k_{l+1}\right)^{-1} V(r, k) \lesssim \int_{1}^{r} t^{-1} V\left(t, k_{\ell+1}\right)^{-1}\left(\int_{\partial \Omega} g^{+}(t, \Phi) d \sigma_{\Phi}\right) d t<\infty \quad(r>1) .
$$

Hence, we can conclude that if $l \geq 1$, then

$$
\mathcal{V}_{l}\left(I_{22}\right)=0
$$

Next, we see $I_{21}$ and note that

$$
0<I_{21}=N\left(H\left(C_{n}(\Omega), l, 0 ; g^{+}\right)\right)(r)-I_{1}^{*}(r)+I_{22}^{*}(r),
$$

where

$$
I_{1}^{*}(r)=\int_{\Omega}\left(\int_{S_{n}(\Omega ;[r, \infty))} g^{+}(Q) K\left(C_{n}(\Omega), l, 0\right)(P, Q) d \sigma_{Q}\right) \varphi_{k}(\Theta) d \sigma_{\Phi}
$$

and

$$
I_{22}^{*}(r)=\int_{\Omega}\left(\int_{S_{n}(\Omega ;[0, r))} g^{+}(Q) K\left(C_{n}(\Omega), l, 0\right)(P, Q) d \sigma_{Q}\right) \varphi_{k}(\Theta) d \sigma_{\Phi}
$$


Since

$$
\left|I_{1}^{*}(r)\right| \leq I_{1}(r) \quad \text { and } \quad\left|I_{22}^{*}(r)\right| \leq I_{22}(r) \quad(r>1)
$$

we see from (3.3) and (3.7) that

$$
\mathcal{V}_{l}\left(\left|I_{1}^{*}\right|\right)=\mathcal{V}_{l}\left(\left|I_{22}^{*}\right|\right)=0
$$

If we can show that

$$
\underset{r \rightarrow \infty}{\limsup } V^{-1}\left(t, k_{l+1}\right) N\left(H\left(C_{n}(\Omega), l, 0 ; g^{+}\right)\right)(r) \leq 0,
$$

we can finally conclude from (3.8) and (3.9) that

$$
\limsup _{r \rightarrow \infty} V^{-1}\left(t, k_{l+1}\right) I_{21}(r) \leq 0,
$$

which gives the required result. To prove (3.10), we recall that $-H\left(C_{n}(\Omega), l, 0 ; g^{+}\right)(P)$ is an a-harmonic function on $C_{n}(\Omega)$ satisfying

$$
\lim _{P \rightarrow Q^{*}, P \in C_{n}(\Omega)}-H\left(C_{n}(\Omega), l, 0 ; g^{+}\right)(P)=g^{+}\left(Q^{*}\right) \leq 0
$$

for any $Q^{*} \in C_{n}(\Omega)$. Hence, we know

$$
-\infty<\mathcal{V}_{l}\left(-H\left(C_{n}(\Omega), l, 0 ; g^{+}\right)\right) \leq \infty
$$

and so

$$
-\infty<\mathcal{V}_{l}\left(H\left(C_{n}(\Omega), l, 0 ; g^{+}\right)\right) \leq \infty
$$

Thus we obtain that if $l \geq 1$, then

$$
\limsup _{r \rightarrow \infty} V\left(t, k_{l+1}\right)^{-1} N\left(H\left(C_{n}(\Omega), l, 0 ; g^{+}\right)\right)(r) \leq 0 .
$$

Therefore,

$$
\mathcal{V}_{l}\left(H\left(C_{n}(\Omega), l, 0 ; g^{+}\right)\right)=0
$$

and so we conclude that

$$
\mathcal{V}_{l}\left(\left|H\left(C_{n}(\Omega), l, 0 ; g^{+}\right)\right|\right)=0
$$

Similarly, we apply the method to $g^{-}$, then we have

$$
\mathcal{V}_{l}\left(\left|H\left(C_{n}(\Omega), l, 0 ; g^{-}\right)\right|\right)=0 .
$$


Since

$$
\left|H\left(C_{n}(\Omega), l, 0 ; g\right)\right| \leq\left|H\left(C_{n}(\Omega), l, 0 ; g^{+}\right)\right|+\left|H\left(C_{n}(\Omega), l, 0 ; g^{-}\right)\right|,
$$

we get the desired conclusion.

Proof of Theorem 1.7 We see from Theorem A that

$$
\begin{gathered}
\limsup _{P \in C_{n}(\Omega), P \rightarrow Q^{*} \in S_{n}(\Omega)} P I_{\Omega}^{a}[g](P)=g\left(Q^{*}\right) \text { and } \\
\limsup _{P \in C_{n}(\Omega), P \rightarrow Q^{*} \in S_{n}(\Omega)} P I_{\Omega}^{a}[|g|](P)=\left|g\left(Q^{*}\right)\right| .
\end{gathered}
$$

Set the two subfunctions on $C_{n}(\Omega)$ as follows:

$$
U_{1}(P)=u(P)-P I_{\Omega}^{a}[g](P) \quad \text { and } \quad U_{2}(P)=u(P)-P I_{\Omega}^{a}[|g|](P)
$$

We have from (1.31) and (3.12)

$$
\limsup _{P \in C_{n}(\Omega), P \rightarrow Q^{*} \in S_{n}(\Omega)} U_{1}(P) \leq 0 \quad \text { and } \quad \limsup _{P \in C_{n}(\Omega), P \rightarrow Q^{*} \in S_{n}(\Omega)} U_{2}(P) \leq 0 .
$$

Hence, it follows from Lemma 2.10 that all of the limits $\mathcal{V}_{p}\left(U_{1}\right), \mathcal{W}_{q}\left(U_{1}\right), \mathcal{V}_{p}\left(U_{2}\right)$ and $\mathcal{W}_{q}\left(U_{2}\right)\left(-\infty<\mathcal{V}_{p}\left(U_{1}\right), \mathcal{W}_{q}\left(U_{1}\right) \leq+\infty, 0 \leq \mathcal{V}_{p}\left(U_{2}\right), \mathcal{W}_{q}\left(U_{2}\right) \leq+\infty\right)$ for any $p, q \in \mathbb{N} \cup\{0\}$ exist. Since

$$
N\left(U_{1}\right)(r)=N(u)(r)-N\left(P I_{\Omega}^{a}[g]\right)(r) \quad \text { and } \quad N\left(U_{2}\right)(r)=N(u)(r)-N\left(P I_{\Omega}^{a}[|g|]\right)(r),
$$

according to Lemma 2.8, we know all of the limits $\mathcal{V}_{p}(u), \mathcal{W}_{q}(u), \mathcal{V}_{p}\left(u^{+}\right)$and $\mathcal{W}_{q}\left(u^{+}\right)$exist and that

$$
\begin{array}{ll}
\mathcal{V}_{p}\left(U_{1}\right)=\mathcal{V}_{p}(u), & \mathcal{W}_{q}\left(U_{1}\right)=\mathcal{W}_{q}(u), \\
\mathcal{V}_{p}\left(U_{2}\right)=\mathcal{V}_{p}\left(u^{+}\right), & \mathcal{W}_{q}\left(U_{2}\right)=\mathcal{W}_{q}\left(u^{+}\right)
\end{array}
$$

for any $p, q \in \mathbb{N} \cup\{0\}$. Since

$$
U_{1}^{+}(P) \leq u^{+}(P)+P I_{\Omega}^{a}[g]^{-}(P),
$$

we have from Lemma 2.8 and (1.32) that

$$
\mathcal{V}_{p}\left(U_{1}^{+}\right) \leq \mathcal{V}_{p}\left(u^{+}\right)<\infty \text { and } \mathcal{W}_{q}\left(U_{1}^{+}\right) \leq \mathcal{W}_{q}\left(u^{+}\right)<\infty
$$

Applying Lemma 2.9 to $U$, we can obtain from (3.13)

$$
u(P) \leq P I_{\Omega}^{a}[g](P)+\sum_{k \in I\left(k_{p+1}\right)} \mathcal{A}_{u}(k) V(r, k) \varphi_{k}(\Theta)+\sum_{k \in I\left(k_{q+1}\right)} \mathcal{B}_{u}(k) W(r, k) \varphi_{k}(\Theta)
$$

for $P=(r, \Theta) \in C_{n}(\Omega)$, which is required. 
Proof of Theorem 1.8 We put $u(P)=h(P)$ and $-h(P)$ in Theorem 1.7, respectively. Then Theorem 1.7 gives the existence of all limits $\mathcal{V}_{p}(h), \mathcal{W}_{q}(h), \mathcal{V}_{p}\left(h^{+}\right), \mathcal{W}_{q}\left(h^{+}\right)$

$$
\mathcal{V}_{p}\left((-h)^{+}\right)=\mathcal{V}_{p}\left(h^{-}\right) \quad \text { and } \quad \mathcal{W}_{q}\left((-h)^{+}\right)=\mathcal{W}_{q}\left(h^{-}\right)
$$

for any $p, q \in \mathbb{N} \cup\{0\}$. Since

$$
\mathcal{V}_{p}(|h|)=\mathcal{V}_{p}\left(h^{+}\right)+\mathcal{V}_{p}\left(h^{-}\right) \quad \text { and } \quad \mathcal{W}_{q}(|h|)=\mathcal{W}_{q}\left(h^{+}\right)+\mathcal{W}_{q}\left(h^{-}\right)
$$

it follows that both limits $\mathcal{V}_{p}(|h|)$ and $\mathcal{W}_{q}(|h|)$ exist. If

$$
\mathcal{V}_{p}(|h|)<\infty \text { and } \mathcal{W}_{q}(|h|)<\infty \text {, }
$$

then we see from (3.14), (3.15) and (1.33) that

$$
\mathcal{V}_{p}\left(h^{+}\right)<\infty, \quad \mathcal{V}_{p}\left((-h)^{+}\right)<\infty, \quad \mathcal{W}_{p}\left(h^{+}\right)<\infty \quad \text { and } \quad \mathcal{W}_{p}\left((-h)^{+}\right)<\infty
$$

Hence, by applying Theorem 1.7 to $u(P)=h(P)$ and $-h(P)$ again, we obtain from (1.34) that

$$
h(P) \leq P I_{\Omega}^{a}[g](P)+\sum_{k \in I\left(k_{p+1}\right)} \mathcal{A}_{h}(k) V(r, k) \varphi_{k}(\Theta)+\sum_{k \in I\left(k_{q+1}\right)} \mathcal{B}_{h}(k) W(r, k) \varphi_{k}(\Theta)
$$

and

$$
h(P) \geq P I_{\Omega}^{a}[g](P)+\sum_{k \in I\left(k_{p+1}\right)} \mathcal{A}_{h}(k) V(r, k) \varphi_{k}(\Theta)+\sum_{k \in I\left(k_{q+1}\right)} \mathcal{B}_{h}(k) W(r, k) \varphi_{k}(\Theta),
$$

respectively, which gives the required result.

Proof of Theorem 1.10 From Theorem 1.4 we have the solution $H\left(C_{n}(\Omega), l, m ; g\right)(P)$ of the Dirichlet problem for the stationary Schrödinger operator on $C_{n}(\Omega)$ with $g$ satisfying (1.28). We consider the function $h-H\left(C_{n}(\Omega), l, m ; g\right)(P)$. Then we see that it is an $a$-harmonic function in $C_{n}(\Omega)$ and vanishes continuously on $\partial C_{n}(\Omega)$. Since

$$
0 \leq\left\{h-H\left(C_{n}(\Omega), l, m ; g\right)\right\}^{+}(P) \leq h^{+}(P)+\left\{H\left(C_{n}(\Omega), l, m ; g\right)\right\}^{-}(P)
$$

for any $P \in C_{n}(\Omega)$,

$$
\mathcal{V}_{l}\left(H\left(C_{n}(\Omega), l, m ; g\right)^{-}\right)=0
$$

and

$$
\mathcal{W}_{l}\left(H\left(C_{n}(\Omega), l, m ; g\right)^{-}\right)=0
$$

from (1.28), (1.36) gives that

$$
\mathcal{V}_{p}\left(\left\{h-H\left(C_{n}(\Omega), l, m ; g\right)\right\}^{+}\right)=0
$$


and

$$
\mathcal{W}_{q}\left(\left\{h-H\left(C_{n}(\Omega), l, m ; g\right)\right\}^{+}\right)=0
$$

\section{From Lemma 2.11 we see that}

$$
h(P)-H\left(C_{n}(\Omega), l, m ; g\right)(P)=\sum_{k \in I\left(k_{p+1}\right)} \mathcal{A}_{h}(k) V(r, k) \varphi_{k}(\Theta)+\sum_{k \in I\left(k_{q+1}\right)} \mathcal{B}_{h}(k) W(r, k) \varphi_{k}(\Theta)
$$

for any $P=(r, \Theta) \in C_{n}(\Omega)$, where $\mathcal{A}_{u}(k)\left(k=1,2, \ldots, k_{p+1}-1\right)$ and $\mathcal{B}_{u}(k)\left(k=1,2, \ldots, k_{q+1}-1\right)$ are all constants. Thus, we obtain the conclusion of Theorem 1.10 .

\section{Competing interests}

The authors declare that they have no competing interests.

\section{Authors' contributions}

$\mathrm{PL}$ carried out the study, participated in the design and drafted the manuscript, GD conceived the study and participated in the design. All authors read and approve the final manuscript.

\section{Acknowledgements}

We wish to express our appreciation to the referee for their careful reading and some useful suggestions which led to an improvement of our original manuscript. Supported by SRFDP (No. 20100003110004) and NSF of China (No. 11071020 and No. 11271045)

Received: 9 February 2012 Accepted: 21 November 2012 Published: 12 December 2012

\section{References}

1. Azarin, VS: Generalization of a theorem of Hayman on subharmonic functions in an $m$-dimensional cone. Transl. Am. Math. Soc. 80(2), 119-138 (1969)

2. Reed, M, Simon, B: Methods of Modern Mathematical Physics, vol. 3. Academic Press, New York (1970)

3. Cranston, M: Conditional Brownian motion, Whitney squares and the conditional gauge theorm. In: Seminar on Stochastic Processes, 1988, pp. 109-119. Birkhäuser, Basel (1989)

4. Cranston, M, Fabes, E, Zhao, Z: Conditional gauge and potential theory for the Schrödinger operator. Trans. Am. Math Soc. 307, 415-425 (1988)

5. Kheyfits, A: Asymptotic behavior of subfunctions of time independent Schrödinger operators. In: Escassut, A, Tutschke, W, Yang, CC (eds.) Some Topics on Value Distribution and Differentility in Complex and P-Adic Analysis. Science Press, Beijing (2008)

6. Levin, B, Kheyfits, A: Asymptotic behavior of subfunctions of the stationary Schrödinger operator. Preprint. http://arxiv.org/abs/math/0211328v1 (2002)

7. Rosenblum, G, Solomyak, M, Shubin, M: Spectral Theory of Differential Operators. VINITI, Moscow (1989)

8. Gilbarg, D, Trudinger, NS: Elliptic Partial Differential Equations of Second Order. Springer, Berlin (1977)

9. Courant, R, Hilbert, D: Methods of Mathematical Phsics, vol. 1. Interscience, New York (1953)

10. Yoshida, H, Miyamoto, I: Solutions of the Dirichlet problem on a cone with continuous data. J. Math. Soc. Jpn. 50(1), 71-93 (1998)

11. Cheng, SY, Li, P: Heat kernel estimates and lower bound of eigenvalues. Comment. Math. Helv. 56, 327-338 (1981)

12. Minakshisundaram, S, Pleijel, A: Some properties of the eigenfunctions of Laplace operator on Riemannian manifolds Can. J. Math. 1, 242-256 (1949)

13. Essén, M, Lewis, JL: The generalized Ahlfors-Heins theorems in certain d-dimensional cones. Math. Scand. 33, 111-129 (1973)

14. Verzhbinskii, GM, Maz'ya, VG: Asymptotic behavior of solutions of elliptic equations of the second order close to a boundary. I. Sib. Math. J. 12(6), 874-899 (1971)

15. Simon, B: Schrödinger semigroups. Bull. Am. Math. Soc. 7, 447-526 (1982)

16. Dinghas, A: Über einen allgemeinen Verzerrungssatz für beschränkte Minimalflächen. Jahresber. Dtsch. Math.-Ver. 69(Heft 3, Abt. 1), 152-160 (1967)

17. Yoshida, $\mathrm{H}$ : Nevanlinna norm of a subharmonic function on a cone or on a cylinder. Proc. Lond. Math. Soc. 54 267-299 (1987)

18. Yoshida, H: Harmonic majorization of a subharmonic function on a cone or on a cylinder. Pac. J. Math. 148(2), 369-395 (1991)

19. Yoshida, H: A type of uniqueness for the Dirichlet problem on a half-spaces with continuous data. Pac. J. Math. 172(2), 591-609 (1996)

20. Yoshida, H, Miyamoto, I: Generalized Poisson integrals on unbounded domains and their applications. Kyoto University Research Information Repository 890, 31-62 (1994). http://hdl.handle.net/2433/84375

21. Qiao, L, Deng, G: A theorem of Phragmén Lindelöf type for subfunctions in a cone. Glasg. Math. J. 53(3), 599-610 (2011) 
22. Bramanti, M: Potential theory for stationary Schrödinger operators: a survey of results obtained with nonprobabilistic methods. Matematiche (Catania) 47(1), 25-61 (1992)

23. Kheyfits, A: Distribution of values and representations of generalized subharmonic functions. Dokl. Akad. Nauk SSSR 314(3), 568-572 (1990). Translation in Sov. Math. Dokl. 42(2), 527-531 (1991)

24. Kheyfits, A: Removable sets, regular boundary points and the Poisson-Jensen formula for generalized subharmonic functions. Dokl. Akad. Nauk SSSR 318(2), 288-290 (1991). Translation in Sov. Math. Dokl. 43(3), 705-707 (1991)

25. Kheyfits, A: The Dirichlet problem in a half-space for the Schrödinger operator with boundary data of arbitrary growth at infinity. Dokl. Akad. Nauk SSSR 325(5), 937-939 (1992). Translation in Dokl. Akad. Nauk, Ross. Akad. Nauk 46(1), 149-152 (1993)

26. Kheyfits, A: Boundary behavior of generalized Poisson integrals for the half-space and the Dirichlet problem for the Schrödinger operator. Proc. Am. Math. Soc. 118(4), 1199-1204 (1993)

27. Kheyfits, A: Dirichlet problem for the Schrödinger operator in a half-space with boundary data of arbitrary growth at infinity. Differ. Integral Equ. 10, 153-164 (1997)

28. Kheyfits, A: Lower estimates of generalized subharmonic functions. Complex Var. Theory Appl. 46(1), 15-30 (2001)

29. Kheyfits, A: Liouville theorems for generalized harmonic functions. Potential Anal. 16(1), 93-101 (2002)

30. Kheyfits, A: Beurling-Nevanlinna inequality for subfunctions of the stationary Schrödinger operator. Proc. Am. Math. Soc. 134(10), 2943-2950 (2006)

31. Levin, B, Kheyfits, A: Asymptotic behavior of subfunctions of the Schrödinger operator in an n-dimensional cone. Dokl. Akad. Nauk SSSR 301(3), 540-543 (1988). Translation in Sov. Math. Dokl. 38(1), 109-112 (1989)

32. Siegel, D, Talvila, EO: Uniqueness for the $n$-dimensional half space Dirichlet problem. Pac. J. Math. 175(2), 571-587 (1996)

33. Yoshida, H, Miyamoto, I: Harmonic function in a cone which vanish on the boundary. Math. Nachr. 202, 177-187 (1999)

34. Qiao, L: Some researches on (generalized) harmonic and superharmonic functions. Dissertation, Beijing Normal University, Beijng (2010)

35. Axler, S, Bourdon, P, Ramey, W: Harmonic Function Theory. Springer, New York (1992)

doi:10.1186/1029-242X-2012-295

Cite this article as: Long and Deng: Some properties for a subfunction associated with the stationary Schrödinger operator in a cone. Journal of Inequalities and Applications 2012 2012:295.

\section{Submit your manuscript to a SpringerOpen ${ }^{\ominus}$ journal and benefit from:}

- Convenient online submission

- Rigorous peer review

- Immediate publication on acceptance

- Open access: articles freely available online

- High visibility within the field

- Retaining the copyright to your article 